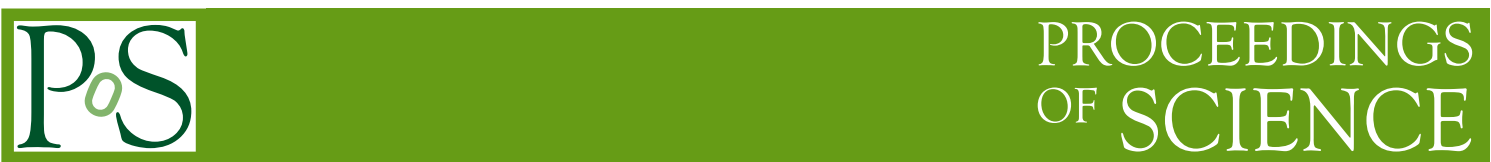

\title{
Improving Higgs predictions with resummation
}

\author{
Frank Petriello* \\ Department of Physics \& Astronomy, Northwestern University, Evanston, IL 60208, USA \\ High Energy Physics Division, Argonne National Laboratory, Argonne, IL 60439, USA \\ E-mail: f-petriello@northwestern.edu
}

\begin{abstract}
Experimental analyses often use jet binning to distinguish between different kinematic regimes and separate contributions from background processes. To accurately model theoretical uncertainties in these measurements, a consistent description of the jet bins is required. We present a complete framework for the combination of resummed results for production processes in different exclusive jet bins, focusing on Higgs production in gluon fusion as an example. We estimate that the relevant theoretical uncertainties on the signal strength in the $H \rightarrow W W^{*}$ analysis are reduced by nearly a factor of 2 compared to the current value using our methodology.
\end{abstract}

Loops and Legs in Quantum Field Theory - LL 2014,

27 April - 2 May 2014

Weimar, Germany

\footnotetext{
* Speaker.
} 


\section{Introduction}

In this contribution we study the resummation of a class of large Sudakov logarithms affecting Higgs boson production in exclusive jet bins at the LHC. These large logarithms occur when the Higgs signal cross section is divided into bins of exclusive jet multiplicity. This division is an experimental necessity; for example, in the zero-jet bin of the WW final state the background is dominated by continuum WW production, while in the one-jet and two-jet bins, top-pair production becomes increasingly important. The optimization of this search requires cuts dependent on the number of jets observed, and therefore also on theoretical predictions for exclusive jet multiplicities.

The theoretical community has invested significant effort in resumming jet-veto logarithms to all orders in perturbation theory in order to more accurately model the LHC Higgs signal. The resummation for the zero-jet bin cross section in the presence of the anti- $k_{T}$ algorithm was first obtained at next-to-leading logarithmic (NLL) accuracy [1] and later extended to NNLL accuracy $[2,3,4]$ using different theoretical approaches. The importance of potentially large $\ln R$ corrections on numerical predictions, where $R$ is the jet-radius parameter in the anti- $k_{T}$ algorithm, was studied in Ref. [5]. A significant reduction of the residual theoretical uncertainties was obtained in the zero-jet bin by resumming the jet-veto logarithms. Given that the theoretical uncertainties are currently one of the largest systematic errors affecting the one-jet bin analyses of the Higgslike particle properties, it is desirable to formulate the resummation when final-state jets are also present.

We begin by considering the phase-space region where the transverse momentum of the hard jet in the exclusive 1-jet bin is much larger than the veto scale. A direct resummation of the Sudakov logarithms using effective field-theory techniques is possible for this choice of parameters. We calculate contributions through next-to-leading order in the exponent of the Sudakov form factor and include the full one-loop functions describing hard, soft, and collinear emissions. This implies that we correctly obtain the first three logarithmic corrections at each order in the QCD coupling constant: $\alpha_{s} L^{2}, \alpha_{s} L$ and $\alpha_{s} ; \alpha_{s}^{2} L^{4}, \alpha_{s}^{2} L^{3}$, and $\alpha_{s}^{2} L^{2} ; \alpha_{s}^{3} L^{6}, \alpha_{s}^{3} L^{5}, \alpha_{s}^{3} L^{4}$; and so on. We have set $L=\ln \left(Q / p_{T}^{\text {veto }}\right)$, where $Q \sim m_{H}$ denotes any hard scale in the problem. We match our results to fixed-order to obtain a NLL' + NLO prediction. We then show how to improve the challenging low $p_{T J}$ region of the 1-jet bin. We do so by using the inclusive 1-jet cross section, supplemented with a fixed-order correction from the 2-jet cross section, to provide a resummed prediction in the low $p_{T J}$ regime. This allows us to present a complete framework for the combination of resummed results for Higgs production. Using this framework, we estimate that the relevant theoretical uncertainties on the signal strength in the $H \rightarrow W W^{*}$ analysis are reduced by nearly a factor of 2 compared to the current value. In this contribution we can only briefly sketch these results. Further details can be found in Refs. $[6,7,8]$.

\section{Review of the formalism}

We use effective-field theory techniques to derive a factorization theorem for exclusive Higgs plus jet production. The factorization of the cross section into separate hard, soft, and collinear sectors is complicated by the presence of the jet algorithm needed to obtain an infrared-safe ob- 
servable. Following the experimental analyses, we use the anti- $k_{T}$ algorithm to define jets. We demand that the final state contain only a single jet with $p_{T J}>p_{T}^{\text {veto }} \sim 25-30 \mathrm{GeV}$. Other jets with a transverse momentum above this threshold are vetoed. Since $p_{T}^{\text {veto }}$ is usually substantially lower than the partonic center-of-mass energy $\left(\lambda \equiv p_{T}^{\text {veto }} / \sqrt{\hat{s}}<<1\right)$, the vetoed observables are usually very sensitive to soft and collinear emissions. We will make the following assumptions in order to proceed in our analysis:

$$
p_{T J} \sim m_{H} \sim \sqrt{\hat{s}} ; \quad 1 \gg R^{2} \gg \lambda^{2} ; \quad \frac{\alpha_{s}}{2 \pi} \log ^{2} R<<1 .
$$

Given that $p_{T}^{\text {veto }} \approx 25-30 \mathrm{GeV}$ and $R \approx 0.4-0.5$, when the leading jet $p_{T J} \sim m_{H}$, the second two assumptions are justified. The first assumption restricts us to specific region of phase space. We will remove that restriction in a later section.

Our effective theory consists of the following low-energy degrees of freedom:

- a collinear jet mode with momentum $p_{J}=\frac{\omega_{J}}{2} n_{J}+k_{J}$, where $n_{J}$ is the light-cone vector along the jet direction;

- two collinear modes propagating along the beam axes $a$ and $b$, with $p_{i}=\frac{\omega_{i}}{2} n_{i}+k_{i}$ for $i=a, b$;

- a soft mode with momentum $k_{s}$.

The residual momenta $k_{J}, k_{i}$ and the soft momentum $k_{s}$ all scale as $\sqrt{\hat{s}} \lambda$, while the large components of the three collinear momenta scale as $\omega_{i} \sim \sqrt{\hat{s}}$. We are able to utilize an effective-theory framework because of how the anti- $k_{T}$ algorithm clusters the soft and collinear modes. The initial clustering combines the final-state hard emissions into a jet, so that the soft radiation sees only the jet direction and does not probe its internal structure. The mixing between the soft and beam sectors is power-suppressed, as is the mixing between the beam and jet sectors. Denoting the measurement function that imposes the jet clustering and vetoing as $\hat{\mathscr{M}}$, these factors imply that we can factor $\hat{\mathscr{M}}$ into the product of measurement functions acting separately on the soft, jet, and beam sectors, up to power-suppressed corrections in $p_{T}^{\text {veto }}$ and $R$. For more details we refer the reader to Refs. [6,7], where the final form for the NLL' cross section is also given.

We briefly comment here on non-global logarithms [9] that first occur at the NLL' level. Although they are not included in our current factorization theorem, to estimate their numerical effect we use the large- $N_{c}$ resummation of these terms derived in Ref. [9]. We include them as a multiplicative correction to our factorization formula. Their numerical effect is small, at or below one percent of the total exclusive Higgs plus one-jet production rate for the relevant values of $m_{H}$ and $p_{T}^{\text {veto }}$. To check the robustness of this result we vary the hard scale appearing in these corrections by a factor of two around their nominal value of $m_{H}$, and find similarly small corrections. We therefore believe that it is numerically safe to neglect these terms in our NLL' result, although they should be further investigated in the future.

\section{Matching NLL' with NLO}

We next match our resummed expression with the fixed-order NLO result to obtain a NLL ${ }^{\prime}+$ NLO prediction. We use the NLO predictions for Higgs plus one-jet contained in MCFM [10]. We 
obtain our prediction by setting

$$
\sigma_{\mathrm{NLL}^{\prime}+\mathrm{NLO}}=\sigma_{\mathrm{NLL}^{\prime}}+\sigma_{\mathrm{NLO}}-\sigma_{\mathrm{NLL}}^{\exp }
$$

In this equation, $\sigma_{\mathrm{NLO}}$ is the fixed-order NLO cross section obtained from MCFM, and $\sigma_{\mathrm{NLL}}^{\prime}$ is the resummed cross section up to $\mathrm{NLL}^{\prime}$ accuracy. $\sigma_{\mathrm{NLL}}^{\exp }$ captures the singular features of $\sigma_{\mathrm{NLO}}$, and is obtained by expanding $\sigma_{\mathrm{NLL}^{\prime}}$ with all scales set to a common value $\mu$. Schematically, we have

$$
\begin{aligned}
& \sigma_{\mathrm{NLL}^{\prime}}=\sigma_{\mathrm{LO}}\left(1+\alpha_{s} g_{0}\right) e^{-L g_{\mathrm{LL}}\left(\alpha_{s} L\right)-g_{\mathrm{NLL}}\left(\alpha_{s} L\right)} \\
& \sigma_{\mathrm{NLL}}^{\exp }=\sigma_{\mathrm{LO}}\left(1+\alpha_{s}\left[-g_{2} L^{2}-g_{1} L+g_{0}\right]\right),
\end{aligned}
$$

where $L g_{\mathrm{LL}}$ and $g_{\mathrm{NLL}}$ resum the leading and next-to-leading logarithms, respectively. The difference between $\sigma_{\mathrm{NLO}}$ and the expanded NLL' result $\sigma_{\mathrm{NLL}}^{\exp }$ only contains power-suppressed contributions for large values of $Q$ :

$$
\sigma_{\text {non-singular }} \equiv \sigma_{\mathrm{NLO}}-\sigma_{\mathrm{NLL}^{\prime}}^{\exp } \sim \mathscr{O}\left(R^{2} L, \frac{p_{T}^{\text {veto }}}{Q} L, \frac{p_{T}^{\text {veto }}}{Q} \log R, \cdots\right)
$$

with $L=\log \left(Q / p_{T}^{\text {veto }}\right)$, and $Q$ stands for any kinematic quantity of order $m_{H}$. Since the scale $Q R$ is used to define the jet mode, the $R^{2} L$ terms are regarded as power suppressed. We have demonstrated explicitly in Ref. [7] that our formalism correctly captures the singular terms at NLO as $L \rightarrow 0$.

\section{Extension to low $p_{T J}$}

As discussed previously, the direct resummation of the exclusive 1-jet bin can only be performed when $p_{T J}$ is much larger than $p_{T}^{\text {veto }}$ and of order $m_{H}$. We therefore divide the low $p_{T J}$ and high $p_{T J}$ regimes using a parameter $p_{T}^{\text {off }}>p_{T}^{\text {veto }}$ :

$$
\sigma_{1}\left(\left[p_{T}^{\text {veto }}, \infty\right] ; p_{T}^{\text {veto }}\right)=\sigma_{1}\left(\left[p_{T}^{\text {veto }}, p_{T}^{\text {off }}\right] ; p_{T}^{\text {veto }}\right)+\sigma_{1}\left(\left[p_{T}^{\text {off }}, \infty\right] ; p_{T}^{\text {veto }}\right) .
$$

We have introduced the following notation to describe the cross sections in different jet bins:

$$
\begin{aligned}
& \sigma_{\mathrm{tot}}: \text { the inclusive cross section, } \\
& \sigma_{0}\left(p_{T}^{\text {veto }}\right): \text { the } 0 \text {-jet cross section, with no jets with } p_{T J}>p_{T}^{\text {veto }}, \\
& \sigma_{\geq 1}\left(p_{T}^{\text {veto }}\right): \text { the inclusive } 1 \text {-jet cross section, with at least } 1 \text { jet with } p_{T J}>p_{T}^{\text {veto }} \\
& \sigma_{1}\left(\left[p_{T a}, p_{T b}\right] ; p_{T}^{\text {veto }}\right): \text { the exclusive } 1 \text {-jet cross section, with } p_{T b}>p_{T J}>p_{T a} \\
& \sigma_{\geq 2}\left(p_{T}^{\text {veto }}\right): \text { the inclusive } 2 \text {-jet cross section, with at least } 2 \text { jets with } p_{T J}>p_{T}^{\text {veto }} .
\end{aligned}
$$

If an event has $p_{T J}<p_{T}^{\text {veto }}$ then it is in the 0 -jet bin. In practice, $p_{T}^{\text {off }}$ is taken to be around $m_{H} / 2$. The second term in this relation can be directly resummed to $\mathrm{NLL}^{\prime}+\mathrm{NLO}$ using the results of Refs. $[6,7]$. To obtain a result for the first term that is improved beyond fixed order by resummation, we note that the following identity can be used to relate the exclusive 1-jet cross section to the inclusive 1-jet and inclusive 2-jet cross sections:

$$
\sigma_{1}\left(\left[p_{T}^{\text {veto }}, p_{T}^{\text {off }}\right] ; p_{T}^{\text {veto }}\right)=\left[\sigma_{\geq 1}\left(p_{T}^{\text {veto }}\right)-\sigma_{\geq 1}\left(p_{T}^{\text {off }}\right)\right]-\left[\sigma_{\geq 2}\left(p_{T}^{\text {veto }}, p_{T}^{\text {veto }}\right)-\sigma_{\geq 2}\left(p_{T}^{\text {off }}, p_{T}^{\text {veto }}\right)\right] .
$$


The first bracketed set of terms give the cross section for one or more jets between $p_{T}^{\text {veto }}$ and $p_{T}^{\text {off }}$. The second bracketed set of terms provides a correction to have only one jet in this $p_{T}$ range. Using the relation in Eq. 4.3, we can construct a (partially) resummed prediction for $\sigma_{1}\left(\left[p_{T}^{\text {veto }}, p_{T}^{\text {off }}\right] ; p_{T}^{\text {veto }}\right)$, as the inclusive 1-jet cross section in the first brackets can be obtained from the resummed 0 -jet cross section, which is known to high accuracy [4]. The inclusive 2-jet cross sections in the second bracket can be calculated at fixed order up to NLO. The difference of inclusive 1-jet cross sections is the same as the following difference of 0 -jet cross sections,

$$
\sigma_{\geq 1}\left(p_{T}^{\text {veto }}\right)-\sigma_{\geq 1}\left(p_{T}^{\text {off }}\right)=\sigma_{0}\left(p_{T}^{\text {off }}\right)-\sigma_{0}\left(p_{T}^{\text {veto }}\right) .
$$

For the resummation of the 0 -jet terms, we use the NNLL'+NNLO results of Ref. [4]. Their difference describes the inclusive 1-jet rate through NLO plus an all-orders series of the inclusive 1-jet logarithms of $p_{T}^{\text {veto }} / m_{H}$ and $p_{T}^{\text {off }} / m_{H}$. The 2-jet terms are calculated at NLO. This means that the leading missing terms in the exclusive 1-jet cross section appear at NNLO and come from the inclusive 1-jet contribution. These corrections can contain at most a single logarithm of the ratios $p_{T}^{\text {veto }} / m_{H}$ and $p_{T}^{\text {off }} / m_{H}$ as well as possibly large nonlogarithmic corrections. However, we will show that the resummation of the 0 -jet terms can capture NNLO 1-jet terms that are known to be large, and our predictions may be tested against the complete $H+1$-jet NNLO cross section once the calculation is complete [11]. There are additional unresummed corrections in the logarithmic series of the exclusive 1-jet cross section induced by the cut on the second jet. These are higherorder terms that convert the resummation from the inclusive to the exclusive 1 -jet cross section (or equivalently correspond to a resummation of the inclusive 2-jet cross sections in Eq. 4.3). These terms enter at $\mathrm{N}^{3} \mathrm{LO}$ and beyond in the exclusive 1-jet cross section, and we will provide evidence that their contribution is small.

To show that Eq. 4.3 improves upon the fixed-order description of the low $p_{T J}$ region, we must demonstrate that a few criteria are fulfilled. First, the contributions from the inclusive 2-jet cross section must be small compared to the 0 -jet terms, so that the resummation can be performed for the bulk of the contribution. Since we will switch to the direct resummation of the 1-jet cross section above $p_{T}^{\text {off }}$, we must also show that the two predictions smoothly match onto each other at that point. Finally, we must demonstrate that these first two points are insensitive to the exact numerical value chosen for $p_{T}^{\text {off }}$. These requirements turn out to be all satisfied; for details, we refer the reader to Ref. [8].

One feature we additionally discuss is that the proposal of Eq. 4.3 represents a systematicallyimprovable framework that can incorporate future theoretical advances as they become available. We therefore include the 0 -jet component of Eq. 4.3 both with and without complex scale setting, which is known to resum large $\pi^{2}$ terms in the $g g \rightarrow H$ form factor relevant for Higgs production $[12,13,14,15,16]$. Similarly, the NNLO hard function (see Ref. [17]) is known to induce a large correction to the gluon-fusion channel of the $H+1$-jet cross section [11]. From these results we can find that for the scale choice $\mu_{R}=\mu_{F}=m_{H}$, the hard function gives a correction to the NLO 1-jet cross section of approximately $30 \%$, compared to a total correction of $40 \%$ when going from NLO to NNLO. It is the dominant source of the NNLO correction for these parameter choices. Furthermore, the enhancement from the hard function is constant over a large range of $p_{T J}$, suggesting that its origin is a large constant term similar to the $\pi^{2}$ corrections that the complex scale setting resums for the 0 -jet cross section. We therefore extend the resummation of Ref. [7] to 
include the NNLO $H+1$-jet hard function. We compare the matching of the low $p_{T J}$ and high $p_{T J}$ regions in two schemes:

- Scheme A: complex scale setting for the 0 -jet terms in the indirect approach, and the direct 1 -jet cross section with the NNLO hard function incorporated.

- Scheme B: no complex scale setting for the 0-jet terms in the indirect approach, and the direct 1 -jet cross section with only the NLO hard function.

Scheme A will lead to larger cross sections due to the $\pi^{2}$ corrections and the NNLO hard function, whose effects on the indirect and direct cross sections are roughly equivalent. Scheme A incorporates more known information than Scheme $\mathrm{B}$, and is therefore our preferred choice for matching the 0 -jet and 1-jet bins. As shown in Ref. [8], it leads to a smoother matching, and that it is also more independent of the parameter $p_{T}^{\text {off }}$ that is used to separate the two regions.

\section{Numerical results}

We now have a consistent framework which incorporates resummation-improved predictions, and accounts for the correlations between their uncertainties, for the 0 -jet and 1-jet bins of Higgs production. We summarize the ingredients below.

- In the 0-jet bin, we use the direct resummation of the cross section as implemented in Ref. [4], currently known to NNLL' + NNLO. We include the effect of complex scale setting in this prediction.

- In the high $p_{T J}$ region of the 1-jet bin, we use the direct resummation of Ref. [7], currently known to NLL' + NLO. We include the NNLO hard function in this prediction.

- In the low $p_{T J}$ region of the 1-jet bin, we use Eq. (4.3). The 0-jet components of this equation are obtained from the 0 -jet resummation, while the inclusive 2-jet pieces are obtained from MCFM at NLO. We note that since we are now able to resum the entire 1-jet cross section and consequently include its dominant corrections beyond NLO, it is consistent to use the NLO result for the inclusive 2-jet piece rather than the LO one.

In this section, we will give the numerical cross sections in each jet bin and their uncertainties. We will compare the results of the resummed predictions to those obtained at fixed order, to demonstrate the improvement obtained. We perform this study for the ATLAS and CMS parameters, taking $m_{H}=125 \mathrm{GeV}$ :

$$
\text { ATLAS: } p_{T}^{\text {veto }}=25 \mathrm{GeV}, R=0.4, \quad \text { CMS: } p_{T}^{\text {veto }}=30 \mathrm{GeV}, R=0.5 .
$$

We use MSTW NNLO PDFs, and assume an 8 TeV LHC.

In Fig. 1, we plot the cross sections in each jet bin against the fixed-order cross sections. The agreement between fixed order and the two matching schemes, as well as the reduced uncertainties when moving beyond fixed order, are clearly visible in the plot. We note that these results, weighted by experimental efficiencies in each jet bin, may directly be used in analyses of the detailed properties of Higgs events. 

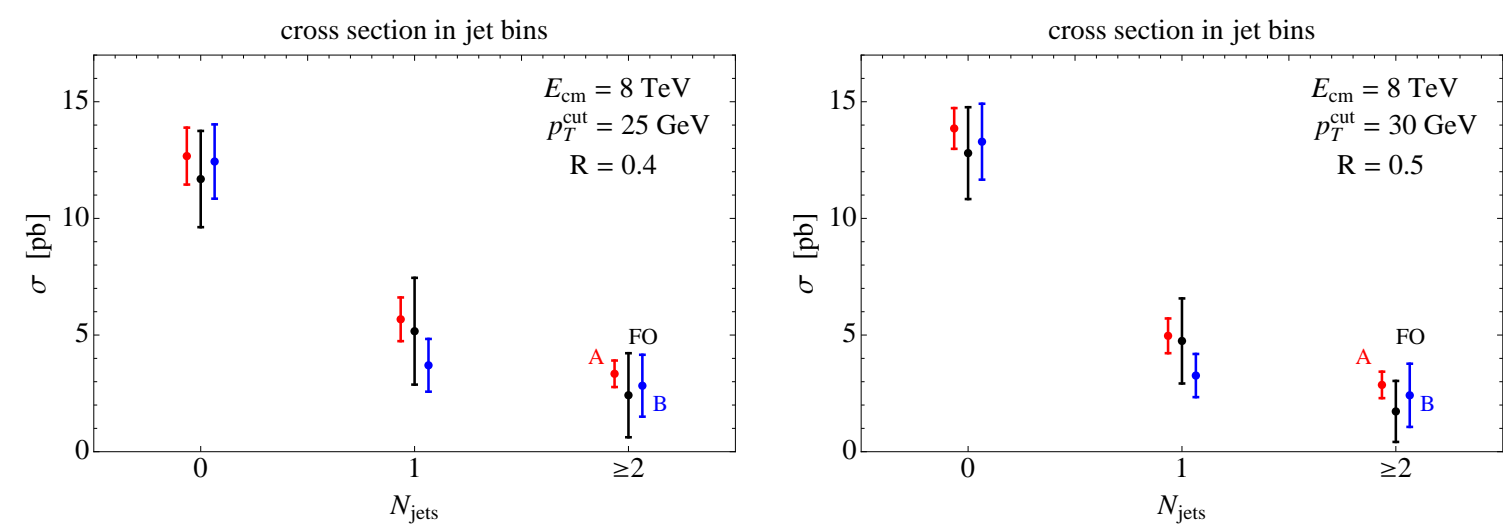

Figure 1: The cross sections in the 0-jet, 1-jet, and inclusive 2-jet bins, for ATLAS (left) and CMS (right) parameter choices. In each jet bin, Scheme A (red left points), Scheme B (blue, right points) are compared to the fixed-order results (black, middle points).

A key measurement in every given production/decay channel is the coupling of the Higgs boson to other Standard Model particles. In the simplest case, this corresponds to a measurement of the signal strength $\mu$, the ratio of the experimentally observed cross section to the SM expectation. For the $H \rightarrow W W^{*}$ decay channel the theoretical uncertainties discussed here, which are those for the expected signal in the sum of jet bins, are a major component of the theory systematics on $\mu$. Using the cross sections and the covariance matrices derived using the procedures sketched here, we can estimate the reduction in theoretical uncertainties on $\mu$ that is gained by using resummed results for the cross sections. More details can be found in Ref. [8]; here we present only the result of this analysis:

$$
\begin{aligned}
& \text { fixed order : } \frac{\Delta \mu}{\mu}=13.3 \%, \\
& \text { Scheme A : } \frac{\Delta \mu}{\mu}=6.9 \% .
\end{aligned}
$$

The ATLAS measurement, using fixed-order results for the cross sections and uncertainties, finds the relative uncertainty on $\mu$ from the expected signal to be $+12 \%$ and $-9 \%$, giving confidence to our estimates. The result in Eq. 5.2 is encouraging, as it shows that the theoretical uncertainties in the expected signal in the determination of $\mu$ are approximately halved by using the results in Scheme A.

\section{Conclusions}

In this work we have presented a complete method for turning the separate resummations of the large logarithms in the 0 -jet and 1-jet bins into a combined covariance matrix for use in experimental studies. A key element of our approach is an extension of the $H+1$-jet resummation into the phase space region where the transverse momentum of the final state jet is small. This is the first result that resums the exclusive 1 -jet cross section across the entire final state phase space. Our approach provides a systematically-improvable framework that can incorporate improved calculations as they become available. As a demonstration, we have shown how adding enhanced 
corrections in the 0 -jet and 1 -jet bins leads to both a smoother matching between the two bins and reduced perturbative uncertainties. Future calculations, such as the NNLO fixed-order result for $H+1$-jet, are easily included in our approach. We find nearly a factor of two reduction in the theoretical uncertainty on the signal strength using our methodology. This is a dramatic improvement in the estimated uncertainty, and will become crucial as the statistical uncertainties on Higgs cross sections continue to decrease during Run II of the LHC. We look forward to the application of our ideas in future LHC analyses.

\section{Acknowledgements}

This work is supported in part by the DOE grants DE-FG02-91ER40684 and DE-AC02-06CH11357.

\section{References}

[1] A. Banfi, G. P. Salam and G. Zanderighi, JHEP 1206, 159 (2012) [arXiv:1203.5773 [hep-ph]].

[2] T. Becher and M. Neubert, JHEP 1207, 108 (2012) [arXiv:1205.3806 [hep-ph]].

[3] A. Banfi, P. F. Monni, G. P. Salam and G. Zanderighi, Phys. Rev. Lett. 109, 202001 (2012) [arXiv:1206.4998 [hep-ph]].

[4] I. W. Stewart, F. J. Tackmann, J. R. Walsh and S. Zuberi, Phys. Rev. D 89, 054001 (2014) [arXiv:1307.1808].

[5] F. J. Tackmann, J. R. Walsh and S. Zuberi, Phys. Rev. D 86, 053011 (2012) [arXiv:1206.4312 [hep-ph]].

[6] X. Liu and F. Petriello, Phys. Rev. D 87, 014018 (2013) [arXiv:1210.1906 [hep-ph]].

[7] X. Liu and F. Petriello, Phys. Rev. D 87, no. 9, 094027 (2013) [arXiv:1303.4405 [hep-ph]].

[8] R. Boughezal, X. Liu, F. Petriello, F. J. Tackmann and J. R. Walsh, Phys. Rev. D 89, 074044 (2014) [arXiv:1312.4535 [hep-ph]].

[9] M. Dasgupta and G. P. Salam, Phys. Lett. B 512, 323 (2001) [hep-ph/0104277].

[10] J. M. Campbell and R. K. Ellis, Nucl. Phys. Proc. Suppl. 205-206, 10 (2010) [arXiv:1007.3492 [hep-ph]].

[11] R. Boughezal, F. Caola, K. Melnikov, F. Petriello and M. Schulze, JHEP 1306, 072 (2013) [arXiv:1302.6216 [hep-ph]].

[12] G. Parisi, Phys. Lett. B 90, 295 (1980).

[13] G. F. Sterman, Nucl. Phys. B 281, 310 (1987).

[14] L. Magnea and G. F. Sterman, Phys. Rev. D 42, 4222 (1990).

[15] V. Ahrens, T. Becher, M. Neubert and L. L. Yang, Phys. Rev. D 79, 033013 (2009) [arXiv:0808.3008 [hep-ph]].

[16] V. Ahrens, T. Becher, M. Neubert and L. L. Yang, Eur. Phys. J. C 62, 333 (2009) [arXiv:0809.4283 [hep-ph]].

[17] T. Gehrmann, M. Jaquier, E. W. N. Glover and A. Koukoutsakis, JHEP 1202, 056 (2012) [arXiv:1112.3554 [hep-ph]]. 